\title{
Hepatozelluläres Karzinom - ein interdisziplinäres Krankheitsbild
}

\author{
Gesprächsleiter: Nisar Peter Malek (Tübingen) Ulf Peter Neumann (Aachen) \\ Jens Ricke (Magdeburg)
}

Teilnehmer:

\author{
Thomas Berg (Leipzig) Christiane Bruns (München) Timm Denecke (Berlin) \\ Heinz-Josef Klumpen (Amsterdam) Hans Lippert (Magdeburg) \\ Maciej Pech (Magdeburg) Markus Peck-Radosavljevic (Wien)
}

\begin{abstract}
Frage 1: Die Erkennung und vor allem die Sicherung des hepatozellulären Karzinoms (HCC) bleiben kontrovers. Die maßgebenden Leitlinien erlauben den klinischen Beweis eines Karzinoms über nichtinvasive Bildgebung. Wie ist der Stellenwert der Leberbiopsie heute und zukünftig?
\end{abstract}

Denecke: Die relevantesten Therapieentscheidungen beim HCC, die von der Diagnosesicherung abhängen, sind die Resektion bzw. Lokalablation und die Lebertransplantation. Hierbei ist, neben der Kenntnis von Lage und Anzahl, die definitive und korrekte Identifizierung der Läsionen als HCC von sensibler Bedeutung. Bei der Gegenüberstellung der bildgebenden Diagnostik und der Biopsie steht die Sensitivität der Verfahren nicht zur Debatte, da nur biopsiert werden kann, was vorher durch die Bildgebung entdeckt wurde. Somit geht es hier um die Artdiagnose einer durch Bildgebung detektierten Läsion. Dabei ist der Verzicht auf eine Tumorbiopsie bei eindeutiger bildgebender Diagnostik von HCCs in Nachteilen der invasiven Biopsie und in der immer zuverlässigeren radiologisch-bildgebenden Diagnostik begründet.

Die Nachteile der perkutanen Biopsie bestehen zum einen in mehreren Risiken, wie z.B. der Blutung oder der Tumorzellverschleppung. Zum anderen ist der negativ prädiktive Wert der Biopsie, auch bei wiederholter Durchführung und trotz bildgeführter Technik, vor allem bei kleinen Tumoren eingeschränkt. Auch ist die feingewebliche Differenzialdiagnostik an Biopsiematerial, das nie die ganze Zielläsion repräsentiert, anspruchsvoll und führt nicht immer zur abschließend korrekten Diagnose. Aufgrund der Praktikabilität können auch nicht alle Kandidatläsionen eines Patienten auf bioptischem Wege gesichert werden.

\section{KARGER}

Fax +497614520714

Information@Karger.com

www.karger.com (c) 2013 S. Karger GmbH, Freiburg

$1662-6664 / 13 / 0292-0113 \$ 38.00 / 0$

Accessible online at:

www.karger.com/vim
Was die Läsionscharakterisierung in der radiologisch-bildgebenden Diagnostik angeht, so gelingt - selbst im kontrastmittelgestützten Ultraschall oder in der Computertomographie $(\mathrm{CT})$ - mit relativ einfachen Kriterien für das Vorliegen eines HCC (d.h. arterielle Hyperkontrastierung und Auswaschphänomen in der Spätphase) in den meisten Fällen eine eindeutige Diagnose. Komplexer, aber bei guter Bildqualität der CT überlegen, ist die Läsionsbeurteilung in der Magnetresonanztomographie (MRT). Eine weitere Verbesserung der MRT-Diagnostik versprechen die hepatozytenspezifischen Kontrastmittel: Die späte Hepatozytenphase liefert einen zusätzlichen Parameter für die Detektion und Charakterisierung von potenziellen HCC-Manifestationen. Mit einer differenzialdiagnostischen Unsicherheit der Bildgebung muss vor allem dann gerechnet werden, wenn im Einzelfall eine technische oder patientenbedingte Einschränkung der Untersuchungsqualität besteht. Ist also die Biopsie von potenziellen HCCLäsionen in Leitlinien durch die bildgebende Diagnostik generell ersetzt, so sollte auch eine konkrete Mindestanforderung an die Untersuchungstechnik und -qualität formuliert bzw. berücksichtigt werden. Zusammengenommen gelingt bei einem Großteil der potenziellen HCC-Läsionen anhand der radiologischen Diagnostik eine zuverlässige Artdiagnose mit geringer Fehlerquote. Dadurch können nach derzeit gängigen Kriterien in der Regel die möglichen Behandlungspfade korrekt gewählt werden, ohne dass eine Biopsie aktuell entscheidende weiterführende Erkenntnisse bringt. Bei nicht eindeutigem Bildbefund oder nicht ausreichend erzielbarer Bildqualität stellt die Biopsie jedoch eine hilfreiche Ergänzung dar.

Peck-Radosavljevic: Die aktuellen Leitlinien der Europäischen Lebergesellschaft (European Association for the Study of the Liver, EASL) zur Diagnostik und Therapie des HCC 
ermöglichen die nichtinvasive, rein radiologische Diagnosestellung eines HCC bei Läsionen über $1 \mathrm{~cm}$ Durchmesser und typischen Anfärbeverhalten in der mehrphasischen CT oder im dynamischen MRT. Der Kontrastmittelultraschall wird in diesen Leitlinien als unzureichend spezifisch nicht akzeptiert. Eine eindeutige Bildgebung ist in größeren Zentren zumindest bei der Hälfte bis zwei Dritteln der HCCs zu erwarten. In diesen Fällen ist die Durchführung einer minimal invasiven Biopsie zu rein diagnostischen Zwecken nicht notwendig. Das Hauptargument dagegen ergibt sich aus dem damit verbundenen Aufwand und dem diagnostischen Grundprinzip, dass Untersuchungen ohne therapierelevanten Mehrwert zu vermeiden sind, und weniger aus der Furcht vor Komplikationen, zumal die in der Literatur angegebenen Komplikationsraten sehr heterogen und in vielen Fällen überzeichnet sind. Klinisch relevante Blutungsepisoden sind bei radiologisch gezielten Biopsien eine Rarität, und über eine zweifelsfrei nachgewiesene und prognoserelevante, Biopsie-assoziierte Tumorzellverschleppung wurde bisher auch kaum berichtet.

Die wahre Stärke der Biopsie liegt jedoch nicht in der simplen Diagnosestellung, sondern in der bisher unersetzlichen Möglichkeit zur Materialgewinnung für eine molekularbiologische Charakterisierung des Tumors zur prognostischen Stratifizierung und gezielten Therapieentscheidung. Eine entsprechende nichtinvasive Tumordiagnostik ist heutzutage für das Mammakarzinom (Hormonrezeptorstatus, HER2/neu), für das Kolonkarzinom (kras-Mutationen) oder das Bronchialkarzinom nicht mehr vorstellbar. Für das HCC war das bis vor kurzer Zeit anders, da trotz erheblicher wissenschaftlicher Bemühungen bisher keine therapierelevante molekulare Klassifizierung des HCC gefunden werden konnte. Allerdings muss an dieser Stelle auch ganz klar festgestellt werden, dass gerade der unglückliche Umstand des häufig fehlenden bioptischen Materials in den letzten Jahren eine molekularbiologische Analyse und potenzielle Subgruppenidentifikation unmöglich gemacht hat. Als besonders bedauerliches Beispiel sei hier die Phase-III-Registrierungsstudie des Multikinaseinhibitors Sorafenib bei fortgeschrittenem HCC erwähnt, in der aufgrund des weitgehend fehlenden Biopsiematerials keine entsprechende Biomarkeranalyse durchgeführt werden konnte. Spätestens mit der Identifizierung des HGF(Hepatozyten-Wachstumsfaktor)-Rezeptors c-MET als eindeutigen prognostischen Faktor für Patienten mit HCC sowie dem klaren Überlebensvorteil durch den Einsatz des sich nun in Phase-III-Entwicklung befindlichen c-MET-Inhibitors Tivantinib wurde jedoch das Ende der nichtinvasiven Diagnostik eingeleitet, zumindest für die palliativen Stadien (BCLC-B, BCLC-C). Da gezielte Therapeutika wie Tivantinib nur in HCCs mit hoher Expression von c-MET im Tumorgewebe wirksam sind, ist für den Einsatz gezielter bzw. personalisierter Therapeutika eine Tumorbiopsie erlässlich. Mit der zu erwartenden Registrierung weiterer Therapeutika mit klarem molekularen Ansatzpunkt wird die Personalisierung auch in der Therapie des HCC deutlich zunehmen und eine bioptische Abklärung voraussetzen, obwohl die bekannten Schwächen der Biopsie wie Stichprobenfehler unwidersprochen sind. Die Fehler der letzten Jahre mit großzügiger, nichtinvasiver Diagnostik in mehreren gescheiterten großen Phase-III-Studien (Sunitinib, Brivanib, Erlotinib, Linifanib), die nun nicht einmal mehr nachträglich molekular für die Identifizierung von Biomarkern von Subgruppen mit einem potenziellen Behandlungsbenefit untersucht werden können, sind nicht mehr wiedergutzumachen und bedeuten schon jetzt einen unwiederbringlichen Wissensverlust über fast ein Jahrzehnt klinischer Hepatomforschung.

Auch wenn es aus radiologischer Sich in vielen Fällen leicht und eindeutig möglich ist, die Diagnose eines HCC nichtinvasiv zu stellen, so ist die minimal invasive gezielte $\mathrm{Bi}$ opsie bereits heute und noch mehr für die Zukunft eine unverzichtbare Voraussetzung für das Management der Patienten und Patientinnen, für die eine aktive Therapie in Betracht kommt.

Frage 2: Entscheidend für die Prognose der Patienten mit HCC ist die Entdeckung in frühen Stadien.

Der überwältigende Anteil der Patienten mit der Diagnose eines HCC in Deutschland wird mit weit fortgeschrittenen Tumoren vorstellig. Wie sieht ein vernünftiges Screening-Programm aus? Welches Vorgehen empfehlen Sie insbesondere niedergelassenen Kollegen und wie definieren Sie Risikopatienten?

Berg: Das HCC eignet sich in besonderer Weise für Früherkennungsstrategien, da die Risikopatienten für die HCCEntwicklung gut charakterisiert sind und die Erkrankung im Frühstadium kurativ zu behandeln ist. Jedoch werden aktuell weniger als $30 \%$ der Patienten in einem HCC-Stadium diagnostiziert, in denen kurative Therapieoptionen zum Einsatz kommen können. Die Gründe für diesen Befund sind vielfältig. Offenbar stellt sich jedoch weniger die Frage, wie, sondern ob Screening- bzw. Surveillance-Untersuchungen bei Personen mit erhöhtem HCC-Risiko zum Einsatz kommen. Risikopatienten für die HCC-Entwicklung werden entweder nicht identifiziert oder nur zu einem geringen Anteil in Überwachungsuntersuchungen eingeschlossen. Nach Statistiken US-amerikanischer Krankenkassen wurden Patienten mit HCC im Vorfeld vor der Diagnosestellung zu nur ca. 40\% von Gastroenterologen/Hepatologen betreut. Eine retrospektive Analyse aus Texas ergab, dass nur 20\% aller Patienten, die sich mit der Diagnose eines HCC in einem Zentrum vorstellten, Screening-Untersuchungen erhalten hatten. Bei der Mehrzahl der Patienten war die chronische Lebererkrankung (20\%) oder die Zirrhose (40\%) nicht bekannt gewesen, oder es wurde kein Screening angeordnet (38\%) bzw. nicht durchgeführt (3\%). Wurden die Patienten von einem Hepatologen betreut, waren die Screening-Raten um das Sechsfache höher und bei Patienten mit Alkoholerkrankung um das Sieben- 
fache geringer. Zu ähnlichen Ergebnissen kommt eine Studie der «National Veterans Administration» bei 1480 mit Hepatitis-C-Virus infizierten Patienten mit HCC. Der Prozentsatz der Patienten mit jährlichen Vorsorgeuntersuchungen (entweder Ultraschall oder Alpha-Fetoprotein (AFP)) betrug $34 \%$. Eine jährliche Surveillance mit AFP und Sonographie bzw. sechsmonatige Intervalluntersuchungen mit mindestens einer Methode (Ultraschall oder AFP) erfolgte nur bei 2 bzw. $6 \%$ aller Patienten. Es ist durchaus wahrscheinlich, dass die US-amerikanischen Erfahrungen in gewissem Umfang auch auf die Situation in Deutschland übertragbar sind.

Die aktuellen europäischen und amerikanischen Leitlinien empfehlen alle 6 Monate die Durchführung von Früherkennungsuntersuchungen bei Patienten mit erhöhtem Risiko allein mittels Ultraschall. Die Serum-AFP-Konzentration als Baustein der nichtinvasiven HCC-Diagnostik wurde in den Leitlinienempfehlungen aufgrund der nur mäßigen Sensitivität und Spezifität dieses Markers aus dem diagnostischen Algorithmus herausgenommen. Prospektive Studien, die die Effektivität des HCC-Screenings bestätigten, haben jedoch sowohl Sonographie als auch AFP-Bestimmungen eingesetzt. Hohe oder im Verlauf langsam ansteigende AFP-Konzentrationen bei Patienten mit chronischen Lebererkrankungen bzw. Zirrhose (ohne aktuellen entzündlichen Schub) sind jedoch ein starkes Indiz für das Vorliegen eines HCC, sodass unter Berücksichtigung der Dynamik dieses Parameters die AFP-Bestimmung aus meiner Sicht weiterhin einen wertvollen Baustein in der Surveillance von Risikopatienten darstellt. Neue HCC-Serummarker wie Descarboxyprothrombin (DCP), glykosyliertes AFP (AFP-L3) oder Osteopontin werden allein oder in Kombination mit AFP evaluiert und könnten in Zukunft $\mathrm{zu}$ einer verbesserten Früherkennung des HCC beitragen.

Eine Verkürzung der Screening-Intervalle auf 3 Monate konnte nicht zu einer Steigerung der HCC-Früherkennungsrate beitragen. Auch bei engmaschigen Kontrolluntersuchungen mittels Ultraschall wird das HCC bei $20-30 \%$ aller Patienten erst in fortgeschrittenen Stadien (außerhalb der MilanKriterien) diagnostiziert. CT oder dynamisches MRT haben sich aufgrund der kostenintensiven Untersuchung sowie der Strahlenbelastung beim CT als Screening-Untersuchung nicht durchgesetzt. Sie sollten jedoch in Erwägung gezogen werden, wenn aufgrund von Adipositas, Darmgasüberlagerung oder anderen technischen Schwierigkeiten die Durchführung einer aussagekräftigen Ultraschalluntersuchung nicht möglich ist oder wenn sich ein kontinuierlicher Anstieg der AFP-Konzentrationen ohne sonographisches Korrelat zeigt.

Alle Patienten mit Leberzirrhose, unabhängig von der Genese der Erkrankung, bei denen potenziell Therapieoptionen bestehen, sollten HCC-Früherkennungsuntersuchungen erhalten. Die chronische Infektion mit dem Hepatitis-B-Virus (HBV) stellt unabhängig von einer Zirrhoseentstehung ein HCC-Risiko dar, sodass Patienten mit HBV-Infektion ebenfalls regelmäßig untersucht werden sollten.
Neuere epidemiologische Studien belegen auch für die nichtalkoholische Fettleberhepatitis (nonalcoholic steatohepatitis, NASH) ein signifikantes Risiko für die HCC-Entwicklung - und das auch bereits in präzirrhotischen Stadien. Liegt zusätzlich ein Diabetes mellitus vor, steigt das HCC-Risiko weiter an. Genetische Polymorphismen im «Patatin-like phospholipase domain containing 3»-Gen (PNPLA 3, Adiponutrin) sind vor allem bei nutritiv-toxischen Lebererkrankungen (alkoholische und nichtalkoholische Fettlebererkrankung) mit einem erhöhten HCC-Risiko assoziiert. Weitere Risikofaktoren für die HCC-Entwicklung auf dem Boden einer chronischen Lebererkrankung sind das männliche Geschlecht, hohes Alter, hoher Body-Mass-Index und ein Nikotinabusus. Anhand dieser Faktoren können Hochrisikopatienten für die HCC-Entwicklung definiert und risikoadaptierte Surveillance-Strategien etabliert werden.

\section{Frage 3: Personalisierte Medizin ist ein Begriff, der viele Diskussionen in der Onkologie bestimmt und nicht nur von Ärzten oder Wissenschaftlern benutzt wird, sondern auch von Pharmakonzernen, Kostenträgern und der Politik. Was bedeutet personalisierte Medizin heute und morgen für das HCC - von der systemischen Therapie bis hin zur Ultima Ratio, der Transplantation? Welche Biomarker haben für Sie heute schon Relevanz?}

Berg: Eine auf bestimmten molekularen Charakteristika des HCC basierende «personalisierte» HCC-Therapie - im klassischen Sinne einer personalisierten onkologischen Medizin - ist für das HCC nicht etabliert. Mit dem MultikinaseInhibitor Sorafenib steht bisher nur eine zugelassene systemische Therapie zur Verfügung, die für alle Patienten mit fortgeschrittenem HCC bei noch ausreichender Leberfunktion empfohlen wird.

Zahlreiche Substanzen, die über zielgerichtete Angriffspunkte insbesondere die Proliferation und Angiogenese des HCC beeinflussen, befinden sich jedoch in der klinischen Entwicklung. Personalisierte Therapiestrategien in Abhängigkeit von molekularen Tumoreigenschaften werden daher in $\mathrm{Zu}$ kunft mit hoher Wahrscheinlichkeit auch für Patienten mit $\mathrm{HCC}$ von Bedeutung sein. Beispiele hierfür stellen die zielgerichtete Therapie gegen Glypican-3 oder die MET-RezeptorTyrosinkinase dar. Glypican-3 wird nur auf etwa 30\% aller HCCs hochexprimiert und reguliert maßgeblich Prozesse des Zellwachstums sowie der Zelldifferenzierung und Migration. Die Effektivität eines rekombinanten humanisierten Antikörpers gegen Glypican-3 scheint nach ersten Analysen wesentlich vom Ausmaß der Glypican-3-Expression abhängig zu sein. Der Tyrosinkinase-Inhibitor Tivantinib führt nach Bindung an den MET-Rezeptor zu Apoptose und verminderter Proliferation und wird im Rahmen von Phase-III-Studien ausschließlich bei Patienten mit hoher MET-Expression evaluiert. 
Stadium und Ätiopathogenese der chronischen Lebererkrankung, auf deren Boden das HCC entstanden ist, haben grundlegende Bedeutung für die Prognose und die Therapieoptionen der Tumorerkrankung. Genexpressionsprofile des den Tumor umgebenden Lebergewebes, nicht jedoch des Tumors selbst, zeigten eine signifikante Korrelation mit der Überlebenswahrscheinlichkeit. Die stadienabhängige Berücksichtigung molekularer Charakteristika der Lebererkrankung könnte somit einen weiteren wichtigen Aspekt im zukünftigen individualisierten Management des HCC darstellen, wird aber auch zu einer Steigerung der ohnehin hohen Komplexität personalisierter onkologischer Therapiekonzepte führen. Eine Sonderstellung im Kontext der personalisierten onkologischen Medizin besitzt das HCC auch dadurch, dass das therapeutische Armamentarium heterogen ist und sich die Relevanz potenzieller Biomarker in Abhängigkeit des Therapieverfahrens (resektiv, lokal ablativ oder systemisch) voneinander unterscheiden wird.

Die Therapie des HCC erfolgt unter Berücksichtigung des Tumorstadiums (insbesondere Zahl und Größe der HCCHerde) und der Leberfunktion. Die Milan-Kriterien stellen den «benchmark» für die Auswahl von Patienten mit HCC für die Lebertransplantation dar. Die AFP-Konzentrationen erlauben darüber hinaus eine Einschätzung des individuellen HCC-Rezidivrisikos und werden bereits als «Biomarker» in Kombination mit den bildgebenden Kriterien für die Entscheidung zur Transplantation berücksichtigt. Wenngleich zahlreiche weitere Faktoren, z.B. das Grading oder der DNAIndex des Tumors, für die Prognoseabschätzung von Relevanz sind und Zahl und Größe nur in begrenztem Umfang die Biologie der Erkrankung reflektieren, hat sich noch kein anderer Marker für die Therapiestratifizierung etablieren können.

Personalisierte Therapiekonzepte sollten jedoch nicht ausschließlich auf eine immer feinere molekulare Charakterisierung der Tumor- bzw. Peri-Tumor-Erkrankung begrenzt werden, sondern sich aus der Synthese aller individuellen patientenbezogenen Befunde entwickeln. Sir William Osler (18491919) formulierte es folgendermaßen: «It is much more important to know what sort of a patient has a disease than what sort of a disease a patient has».

Bruns: Personalisierte Medizin bedeutet beim HCC ähnlich wie bei anderen Krebserkrankungen eine individuell auf das onkologische Risiko des jeweiligen Patienten «maßgeschneiderte» Therapie unter Berücksichtigung genereller Faktoren wie Alter des Patienten, Komorbiditätsprofil, Lebensqualität bis hin zu Patientenwunsch.

Die Lebertransplantation ist die onkologisch beste Therapie für das auf die Leber begrenzte HCC, da durch die Transplantation nicht nur die radikalste Resektion des Tumors, sondern auch zusätzlich die Entfernung des präneoplastischen Gewebes ermöglicht wird. Die Organverfügbarkeit sowie die aktuellen Allokationsrichtlinien (BCLC, AASLD oder EASL
Guidelines) sind allerdings entscheidende Einflussgrößen der «maßgeschneiderten» Therapie für die Patienten, deren Erkrankungsstadium optimalerweise mit antizipiertem, bestmöglichem Langzeitergebnis durch Lebertransplantation behandelt werden könnten [1,2]. Um während der entsprechend langen Wartezeit eine Tumorprogression zu vermeiden, erhalten diese Patienten eine neoadjuvante Überbrückungstherapie (Bridging). Hier steht eine Vielzahl von Bridging-Verfahren zur Verfügung, die stadienadaptiert, aber auch komplementär eingesetzt werden können. Lokoregionär ablative Verfahren (transarterielle Chemoembolisation, TACE), selektive interne Radiotherapie (SIRT), Radiofrequenzablation (RFA), stereotaktische Bestrahlung (Cyberknife), systemische Therapien mit dem Multityrosinkinase-Inhibitor Sorafenib oder auch die chirurgische Resektion können zur lokalen Tumorkontrolle während der Wartezeit eingesetzt werden und sind damit ein Teil des «maßgeschneiderten» Therapieansatzes beim HCC.

Die onkologische Wertigkeit aller neoadjuvanten BridgingAnsätze ist bis dato nur unzureichend geklärt, insbesondere, ob sich dadurch die Tumorbiologie oder das Metastasierungsverhalten über den Zeitraum der Therapie oder nach Transplantation verändert. Entscheidend ist, dass i) dem Patient durch Ansprechen auf eine neoadjuvante Therapie der $\mathrm{Zu}$ gang zur Lebertransplantation überhaupt ermöglicht wird und dass ii) Therapieansprechen als positiver Prognoseparameter bei günstiger Tumorbiologie gewertet wird. Im eigenen Krankengut korreliert das Überleben nach Transplantation mit dem Response-Grad nach RECIST. Demgegenüber wird zunehmend evidenter, dass die momentan basierend auf Tumorgröße und -anzahl geltenden Milan-Kriterien für den individuellen Patienten keine prognostische Wertung zulassen. Retrospektive Patientenanalysen legen nahe, dass HCCs, die durch neoadjuvante Therapien ein Downstaging innerhalb der Milan-Kriterien erfahren haben, eine ähnlich gute Prognose aufweisen wie Tumoren, die bei Diagnosestellung bereits innerhalb der Milan-Kriterien waren [3-5].

Weitere prädiktive oder prognostische Biomarker, die stadiengerechte, «maßgeschneiderte» Therapiekonzepte beim HCC relevant beeinflussen, gibt es bislang nicht.

Klumpen: Personalisierte Medizin ist das Zusammenspiel von Faktoren, die sich auf drei Hauptfaktoren beziehen: i) der Tumor, ii) der Patient, iii) die Behandlung. Bei der Aufklärung der ersten zwei Faktoren werden uns, neben retrospektiven Analysen, größere genetische Studien sicherlich weiterhelfen auf der Suche nach den richtigen Zielpunkten von Medikamenten und den Patienten, die den meisten Nutzen von einer Behandlung haben werden. Für den dritten Punkt will ich mich auf die medikamentöse Behandlung konzentrieren. Hierbei ist es wichtig zu wissen, dass ein Medikament neben dem richtigen Wirkungsmechanismus auch die richtige Dosis im Patienten erreichen muss, um optimal $\mathrm{zu}$ wirken. Für andere Medikamente sowie Antibiotika, Antidepressiva 
und Immunsuppressiva haben wir uns schon lange daran gewöhnt, dass dies wichtig ist, um einen bestimmten Blutspiegel zu erreichen. Das Einstellen auf den richtigen Blutspiegel erhöht den Nutzen der Behandlung und verhindert eine Überoder Unterbehandlung, auch bei Krebsmedikamenten, und wird auch «therapeutic drug monitoring (TDM)» genannt [6]. Für das HCC und die Behandlung mit Sorafenib ist noch nicht deutlich, was der richtige Blutspiegel sein sollte, um eine Über- oder Unterbehandlung zu verhindern. Die empfohlene Dosis ist für alle Patienten die gleiche $(2 \times 400 \mathrm{mg}$ pro Tag $)$. Eine Anpassung dieser Dosis nach unten wird in der Praxis nur bei Unverträglichkeit angewandt. Eine Dosissteigerung bei dem Ausbleiben von Nebenwirkungen und fehlender Wirksamkeit ist nicht gebräuchlich, wäre jedoch logisch und sicherlich darin begründet, dass die Ausscheidung von Sorafenib im Laufe der Zeit zunimmt [7].

Für ein anderes Medikament, Imatinib, auch ein Tyrosinkinase-Inhibitor wie Sorafenib, sind die oben beschriebenen Phänomene bekannt, und es wird das Prinzip des TDM in der Klinik empfohlen [8, 9].

Genau wie bei Imatinib ist es auch bei Sorafenib so, dass eine große Inter-Patienten-Variabilität besteht, was den Blutspiegel bei gleicher Dosierung betrifft. Im Moment laufen verschiedene Studien, um den Zusammenhang zwischen dem Blutspiegel bei Sorafenib mit der Wirkung des Medikamentes zu korrelieren [10]. Komplizierend kommt hinzu, dass Sorafenib im Individuum in mehr oder weniger aktiven Metaboliten verschieden metabolisiert wird. Die Verteilung der Menge der Metaboliten ist von verschiedenen Faktoren abhängig, wie z.B. der Leberfunktion und der Komedikation. Es könnte also der Fall sein, dass nicht allein der Blutspiegel von Sorafenib selbst, sondern auch der Blutspiegel der Metaboliten wichtig ist, um die optimale Behandlung, also die personalisierte Medizin, zu erreichen. Davon ausgehend, dass wir innerhalb der nächsten Zeit wissen werden, bei welchem Tumor die besten Wirkungserfolge mit Sorafenib erzielt werden können und welchen Blutspiegel wir erreichen sollten, haben wir leider unser Ziel noch nicht erreicht. Es gibt noch das Problem der Verträglichkeit. Manche Patienten werden die Behandlung nicht vertragen, da ihr Metabolismus, möglicherweise genetisch bedingt, nicht die gewünschten Blutspiegel ohne unverträgliche Nebenwirkungen erreichen lässt.

Zusammenfassend sollte bei einer personalisierten Medizin neben der Individualisierung abhängig vom Tumor und den Patientenumständen auch die richtige Zieldosis bei der medikamentösen Behandlung bekannt sein. Die Resultate von laufenden Studien werden uns hierbei hoffentlich bald weiterhelfen.

Pech: Klassische Biomarker nutzen wir derzeit so gut wie nicht für die Indikationsstellung oder Durchführung einer interventionell-radiologischen Behandlung des HCC. Einen Stellenwert haben aber umgekehrt interventionelle Eingriffe als eigenständige Biomarker bzw. prognostische Faktoren.
Herr Professor Otto aus Mainz beispielsweise hat in früheren Arbeiten gezeigt, dass das Ansprechen auf eine Chemoembolisation als Bridging zur Transplantation sehr gut mit der Prognose der Patienten korreliert - Chemoembolisation dient hier also potenziell als Stratum für die Transplantationsindikation. Zukünftig von Wichtigkeit auch für minimal invasive Eingriffe könnte die Bestimmung von Serummarkern wie VEGF (vaskulärer endothelialer Wachstumsfaktor) oder anderen werden. Wir wissen mittlerweile, dass Bestrahlung oder Chemoembolisation der Leber angiogenetische Kaskaden eröffnen. VEGF hat in multiplen Studien einen negativen prognostischen Wert bewiesen. Derzeit ist nicht vorhersagbar, welcher Patient nach einer RFA, Bestrahlung oder Chemoembolisation einen VEGF-Anstieg erleidet; zukünftig wird es spannend sein, ob eine Unterbrechung der VEGF-Kaskaden oder anderer Angiogenese-Pfade durch spezifische Moleküle die Prognose der Patienten verbessert. Ansonsten verstehe ich den Begriff «personalisierte Medizin» eher als einen Vorgriff auf die Individualisierung der Therapiekonzepte und vor allem der Sequenzen des Werkzeugeinsatzes. Wir sehen sehr häufig, dass wir Patienten über Monate und Jahre mit unterschiedlichen Verfahren - sei es lokal, lokoregionär oder systemisch - behandeln, die sequenziell eingesetzt bzw. beim Restaging zum Follow-up nach beispielsweise 3 Monaten immer wieder neu entschieden werden. Ich glaube persönlich, dass die größten Prognosevorteile für das HCC auf dem Wege der perfekten Sequenzierung der Therapiemaßnahmen für individuelle Krankheitsentwicklungen zu erzielen sind.

\section{Frage 4: Die Lebertransplantation ist die einzige definitive Therapie des HCC in Zirrhose. Wo sehen Sie die Lebertransplantation in zehn Jahren?}

Bruns: Bislang gilt die Lebertransplantation als optimalste Therapie für Patienten mit hepatisch begrenzten HCCs. Diese Patienten erhalten zur Überbrückung der teilweise langen Wartezeit im Eurotransplant-Raum zur Verhinderung der Tumorprogression eine neoadjuvante Bridging-Therapie. Die jeweils beste Therapiemodalität wird bislang unter Berücksichtigung der Tumorlast, der Tumorlokalisation und des Allgemeinzustands des Patienten in einem interdisziplinären Tumorboard festgelegt.

Patienten mit Tumoren innerhalb der Milan-Kriterien werden Extrapunkte, d.h. Standard Exception (SE) Points, zugebilligt, wodurch in der Regel nach 12-18 Monaten über die reguläre Organvergabe eine Lebertransplantation ermöglicht wird. Ausgehend von einem initialen match-MELD (Model for End-Stage Liver Disease) von 22 Punkten, der einer 3-Monats-Mortalität von $15 \%$ entspricht, werden die Patienten alle 3 Monate um den Punktewert einer 3-MonatsMortalität von $10 \%$ hochgestuft. Dabei ist bislang Folgendes zu berücksichtigen: i) Tumoren müssen vor der Listung je- 
derzeit innerhalb der Milan-Kriterien gelegen sein; ii) Tumoren, die nach Ansprechen auf eine neoadjuvante BridgingTherapie die Milan-Kriterien erreichen, erhalten keine SEZusatzpunkte; iii) Tumoren müssen auch zum Zeitpunkt der Höherstufung in 3-Monats-Schritten immer die Milan-Kriterien erfüllen. Patienten mit Tumoren außerhalb der MilanKriterien erhalten selbst bei gutem Ansprechen auf eine neoadjuvante Therapie (auch bei Rückbildung bis innerhalb der Milan-Kriterien) keine Sonderpunkte und sind so trotz teilweise guter Prognose im Wesentlichen auf eine LeberLebendspende angewiesen.

Ein Fortschritt in der Lebertransplantation bei zugrunde liegendem HCC wäre einerseits die Weiterentwicklung in Richtung individualisierter, Biomarker-gestützter BridgingVerfahren, um eine «maßgeschneiderte» neoadjuvante Therapie mit hoher Wahrscheinlichkeit einer Tumorresponse für jeden gelisteten Patienten zu erzielen.

Auch ist vorstellbar, dass die Response-Evaluation bei «maßgeschneiderter» neoadjuvanter Therapie zukünftig durch die Untersuchung molekularer Marker oder den Einsatz von molekularem Imaging verfeinert und wohlmöglich frühzeitiger erfassbar ist.

In ähnlicher Weise könnte ein Biomarker-unterstütztes onkologisches Risikoprofil unter Einbeziehung moderner Techniken zur Response-Evaluation und der Zeit der Krankheitsstabilität die bisherigen Allokationsrichtlinien zur Transplantation bei HCC ergänzen.

Es gibt bereits erste Initiativen in den USA und in Deutschland, Patienten mit nachgewiesener Tumorresponse auf neoadjuvante Therapien den Zugang zu einer Lebertransplantation im regulären Verteilungsverfahren zu erleichtern.

Berücksichtigt man die bleibende Organknappheit, ist ein Ausbau der Forschung im Bereich des «tissue engineerings» zur funktionellen Unterstützung marginaler Organangebote denkbar, wobei derartige Ansätze bei onkologischen Grunderkrankungen kritisch bleiben [11].

Lippert: Das HCC entsteht in der Zirrhose. Dies kann sowohl solitär als auch multilokulär sein. Hier lassen sich nur individuelle Konzepte umsetzen, die die Grunderkrankung, den Gesamtzustand und die lokale Situation einbeziehen. Mit einer Lebertransplantation wird das Organ mit dem Tumor entfernt. Dennoch werden Rezidive nach einer Lebertransplantation beschrieben. Die Milan-Kriterien sind heute die tragfähigste Entscheidungshilfe, weil sie Transplantationskandidaten potenziell auf heilbare, frühere Stadien reduzieren. Ich gehe davon aus, dass sich daran nichts ändern wird.

Schließt man die aktuellen Probleme der Transplantationsmedizin ein, so ist vor allem die kurzfristige Verfügbarkeit von Organen ein wesentlicher Faktor, der das Handeln bei frühen HCCs bestimmt. Das Bridging mittels Resektion, lokaler ablativer Therapie und einer Minderung der kanzerogenen Gefahr in der Zirrhoseleber ist mittlerweile und sicher auch weiterhin Standard.
Systematische Verbesserungen sind durch eine profunde medikamentöse Prophylaxe, individuelle Entscheidungen zu sparsamerer Resektion und hochpräzise Lokaltherapien im Sinne eines Downstagings über die nächsten 20 Jahre noch denkbar. Die Leber-Lebendspende kann die Organknappheit erheblich positiv beeinflussen. Keine höhere Effektivität durch eine Transplantation sehe ich bei cholangiozellulärem Karzinom.

\section{Teilnehmer}

Prof. Dr. med. Thomas Berg

Sektion Hepatologie, Department für Innere Medizin,

Neurologie und Dermatologie

Medizinische Klinik und Poliklinik für Gastroenterologie und Rheumatologie

Universitätsklinikum Leipzig

Liebigstraße 20, 04103 Leipzig, Deutschland

thomas.berg@medizin.uni-leipzig.de

Prof. Dr. med. Christiane Bruns

Onkologische Chirurgie, Chirurgische Klinik und Poliklinik

Klinik für Allgemeine, Viszeral-, Transplantations-, Gefäß- und

Thoraxchirurgie

LMU München - Campus Großhadern

Marchioninistraße 15, 81377 München, Deutschland

Christiane.Bruns@med.uni-muenchen.de

PD Dr. med. Timm Denecke

Klinik für Radiologie

Charité - Universitätsmedizin Berlin, Campus Virchow-Klinikum Augustenburger Platz 1, 13353 Berlin, Deutschland timm.denecke@charite.de

Dr. Heinz-Josef Klumpen

Medizinische Onkologie, Academisch Medisch Centrum

Universität von Amsterdam

Meibergdreef 9, 1105AZ Amsterdam, Niederlande

h.klumpen@amc.nl

Prof. Dr. med. Hans Lippert

Klinik für Allgemein-, Viszeral- und Gefäßchirurgie Universitätsklinikum Magdeburg A.ö.R.

Leipziger Straße 44, Haus 10, 39120 Magdeburg, Deutschland hans.lippert@med.ovgu.de

Prof. Dr. med. Maciej Pech

Klinik für Radiologie und Nuklearmedizin Universitätsklinikum Magdeburg AöR

Leipziger Straße 44, 39120 Magdeburg, Deutschland maciej.pech@med.ovgu.de

Ao. Univ. Prof. Dr. Markus Peck-Radosavljevic Abteilung Gastroenterologie und Hepatologie Medizinische Universität Wien Währinger Gürtel 18-20, 1090 Wien, Österreich markus.peck@meduniwien.ac.at 


\section{Literatur}

1 European Association For The Study Of The Liver; European Organisation For Research And Treatment Of Cancer: EASL-EORTC clinical practice guidelines: management of hepatocellular carcinoma. J Hepatol 2012;56:908-943.

2 Bruix J, Sherman M: Management of hepatocellular carcinoma: an update. Hepatology 2011;53: 1020-1022.

3 Otto G, Herber S, Heise M, et al: Response to transarterial chemoembolization as a biological selection criterion for liver transplantation in hepatocellular carcinoma. Liver Transpl 2006;12: 1260-1267.

4 Ravaioli M, Grazi GL, Piscaglia F, et al: Liver transplantation for hepatocellular carcinoma: results of down-staging in patients initially outside the Milan selection criteria. Am J Transplant 2008; 8:2547-2557.
5 Yao FY, Kerlan RK Jr, Hirose R, et al: Excellent outcome following down-staging of hepatocellular carcinoma prior to liver transplantation: an intention-to-treat analysis. Hepatology 2008;48:819-827. 6 Gao B, Yeap S, Clements A, Balakrishnar B, Wong M, Gurney H: Evidence for therapeutic drug monitoring of targeted anticancer therapies. J Clin Oncol 2012;30:4017-4025.

7 Arrondeau J, Mir O, Boudou-Rouquette P, et al: Sorafenib exposure decreases over time in patients with hepatocellular carcinoma. Invest New Drugs 2012;30:2046-2049.

8 Teng JF, Mabasa VH, Ensom MH: The role of therapeutic drug monitoring of imatinib in patients with chronic myeloid leukemia and metastatic or unresectable gastrointestinal stromal tumors. Ther Drug Monit 2012;34:85-97.
9 Judson I: Therapeutic drug monitoring of imatinib - new data strengthen the case. Clin Cancer Res 2012;18:5517-5519.

10 Ricke J, Schuette K, Rosmorduc O, et al: Sorafenib in combination with local microtherapy guided by gadolinium-EOB-DTPA enhanced MRI in patients with inoperable hepatocellular carcinoma (HCC) (SORAMIC). ASCO Meeting Abstracts 2012;30(15s):TPS4148.

11 Török E, Lutgehetmann M, Bierwolf J, Melbeck S, Düllmann J, Nashan B, Ma PX, Pollok JM: Primary human hepatocytes on biodegradable poly(l-lactic acid) matrices: a promising model for improving transplantation efficiency with tissue engineering. Liver Transpl 2011;17:104-114. 